\title{
SELECTIVE ELECTROCHEMICAL RELEASE ETCHING OF EUTECTICALLY BONDED MICROSTRUCTURES
}

\author{
H. Gradin*, S. Braun, M. Sterner, G. Stemme and W. van der Wijngaart \\ Microsystem Technology Lab, KTH - Royal Institute of Technology, Stockholm, Sweden
}

\begin{abstract}
This paper reports on the successful demonstration of a novel microfabrication method in which eutectic gold bonded microstructures are selectively electrochemically release etched. This method offers several advantages: both a strong permanent bond and a temporary bond is achieved on the same die, the footprint of the temporary bonded structures is allowed to be larger than the footprint of the permanently bonded structures and the used etchants provide a larger process compatibility than the etchants of other release etch methods. Eutectically bonded $350 \mu \mathrm{m}$ wide silicon structures were fully released after 1 hour of electrochemical etching followed by 1.5 hours wet etching of the TiW adhesion layer.
\end{abstract}

\section{KEYWORDS}

Wafer bonding, eutectic bonding, electrochemical etching, release etching, selective etching

\section{INTRODUCTION}

In MEMS different machined or non-machined substrates can be combined with each other using wafer-towafer bonding technologies [1], which allow the fabrication and packaging of complex three-dimensional (3D) microcomponents. Such microcomponents often feature moving structures which must be detached from their underlying substrate at the end of the fabrication. Using wafer-to-wafer bonding technology, there are basically two methods to allow for a moving structure. The first method is the selective bonding of areas to be bonded while avoiding the bonding of the moving structures to the underlying substrate. The second method is a bond-and-release approach, which is a full wafer bond followed by subsequent removal of the bond interface material underneath the structures to be released.

Selective bonding of two substrates is obtained with two different principles. The first principle is to pattern the bond interface material prior to bonding, defining bonding and non-bonding areas. Examples of patterned bond interface layers are adhesive layers only applied on areas where bonding is desired $[1,2]$ as well as bond blocking layers such as gold or platinum to define local non-bonding areas [3]. The second principle is to use elevated temperature bonding methods and to localize the heat to the desired areas of the bond interface. Examples of this approach are integrated heaters for both selective eutectic and silicon fusion bonding [4]. In selective bonding, the non-bonded parts must remain mechanically connected to the bonded parts by mechanical supports during processing. The release of the moving structure by break-away of the MEMS support structures through dicing or through controlled fracture has been shown [5]. However, the mechanical supports necessary in this method limit the freedom in the MEMS design and potentially increase the footprint of the device. Also, sensitive moving structures can be destroyed during dicing of the mechanical supports.

In a bond-and-release scheme, all structures are bonded and the structures to be released are separated in a release step at a later stage of the fabrication. This method is based on wafer-bonding methods with intermediate bonding layers which can be sacrificially etched with a high selectivity. Examples of intermediate bonding materials serving as sacrificial etch layer are oxide [6] and polymers [1, 7]. However, the oxide sacricifical layer is wet etched using aggressive hydrofluoric (HF) acid based etchants, which is incompatible with many MEMS materials [8]. The polymer sacrifical layers are commonly etched in an oxygen plasma ashing process, which can heat up and oxidize the MEMS component and possibly affect the performance of the device.

In general, sacrificial release etching requires the release bond area to be substantially smaller than the permanent bond area i.e. the release selectivity is dependent on the geometry of the structure. To release a structure with larger bond area than a structure which should remain bonded, additional etch holes are usually introduced which reduce the underetch dimensions and thereby reduce the time necessary to fully underetch the structure to be released. However, the etch holes restrict the design freedom and may decrease the performance of the layers to be detached. This issue is addressed by selectively dissolving a polymer bond using laser ablation [9]. However, this technology is limited to a bond substrate that is transparent to the laser wavelength and a bond polymer that strongly absorbs the laser light.

The current paper presents a method to permanently bond all structures using a strong eutectic Au-Si wafer bond [10], followed by selective electrochemical etching [11] of the bond interface material formed during the eutectic bonding, which allows to detach selected structures. Electrochemical release of microstructures has been shown before [12], however, only for the detachment of layers on a deposited sacrificial aluminum layer and not in conjunction with wafer bonding. 


\section{SELECTIVE BOND AND RELEASE SCHEME}

The presented method is based on eutectic gold silicon bonding of two substrates [10]. The eutectic bonding offers a strong bond at relatively low bond temperatures and is formed above the eutectic point of $363{ }^{\circ} \mathrm{C}$ for the $\mathrm{Au}-$ $\mathrm{Si}$ composition. The eutectic at this temperature contains 19 atomic $\%$ silicon.

After the eutectic bonding of all structures, the bond interface material formed during the eutectic bonding is electrochemically etched by placing the MEMS device in chloride salt solution and applying a potential between the bond interface material and a counter electrode. This etching method provides the advantages of high material compatibility as well as a high etch selectivity.

To release the selected structures, their underlying bond interface material must be electrically connected to the etch potential. At the same time, to ensure that the remaining structures remain permanently bonded, their underlying bond interface material must be electrically disconnected from the etch potential.

\section{FABRICATION OF FIRST TEST STRUCTURES}

Figure 1 illustrates the fabrication scheme developed in this work. First two different substrates were prepared. On a glass wafer (for visual inspection) a $60 \mathrm{~nm}$ thick TiW adhesion layer and a $500 \mathrm{~nm}$ thick gold layer were sputterdeposited (Fig. 1a). Thereafter the gold layer and the TiW layer were patterned to form electrical connections between all structures to be released and to electrically isolate the structures not to be released. To avoid an unwanted electrical connection between the structures, the TiW/gold layer must be deposited on a dielectric layer, which in the presented work was provided by the glass substrate.

The other substrate must have a silicon layer to allow for the eutectic Au-Si formation. A $300 \mu \mathrm{m}$ thick $<100>$ silicon wafer (Fig. 1b) was therefore used. The wafer was patterned using deep reactive ion etching (DRIE). Subsequently, the wafer was prepared for the eutectic bonding by sputter etch removal of the native oxide, followed by in-situ sputter deposition of $500 \mathrm{~nm}$ gold directly on the silicon.

The two substrates were aligned with the gold sides facing each other and bonded for $20 \mathrm{~min}$ in vacuum at $400{ }^{\circ} \mathrm{C}$ and 1 bar load pressure (Fig. 1c). Next, a second DRIE on the top side silicon side was performed to create freestanding, eutectically bonded silicon structures (Fig. 1d). The free standing structures ensures that there will be no current path between the silicon structures that should remain bonded and the ones to be released.

For the selective electrochemical release etch (Fig. 1e), single chips were diced out and placed in degassed $5 \mathrm{M}$ $\mathrm{NaCl}$ electrolyte. A probe needle was used to contact the patterned TiW layer and the bond interface material under-

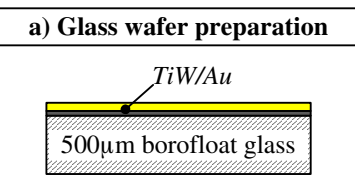

Sputter-deposition of $60 / 500 \mathrm{~nm}$ TiW/Au on borofloat glass wafer

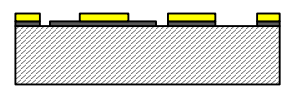

Patterning of $\mathrm{Au}$ and TiW to electrically isolate the structures NOT to be released

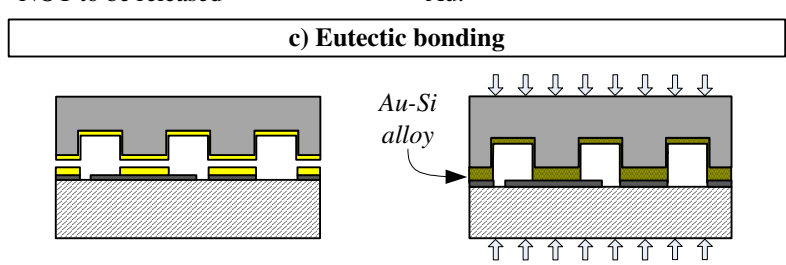

Alignment and subsequent bonding of the stack at $400{ }^{\circ} \mathrm{C}$ and 1 bar applied load pressure for $20 \mathrm{~min}$.

d) Top side free etch

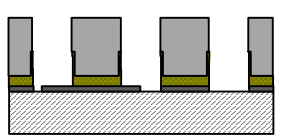

Second DRIE to free etch the Si structures.

e) Selective electrochemical release etching

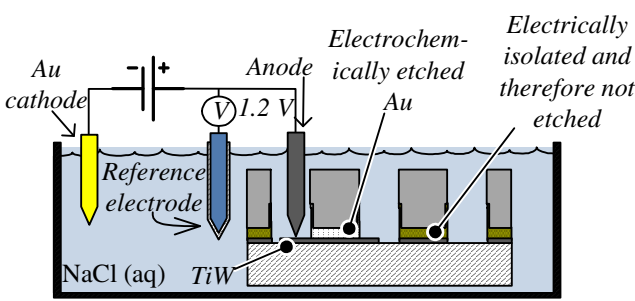

Selective electrochemical etching of the contacted $\mathrm{Au}$ in the bonding layer.

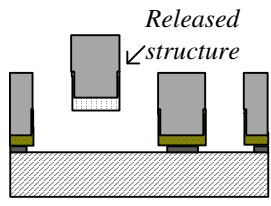

Etching if TiW layer using $\mathrm{H}_{2} \mathrm{O}_{2}$ to detach the selected structures.

Figure 1: Fabrication

neath the silicon structures to be released, while the other structures remain electrically isolated. The bond interface material to be removed in the eutectic Au-Si bond was coupled as anode of the electrochemical cell and a gold electrode was used as counter electrode. A constant voltage of $1.2 \mathrm{~V}$ versus an $\mathrm{Ag} / \mathrm{AgCl} \mathrm{KCl}$ saturated reference electrode was applied to drive the electrochemical etching of the connected gold alloy layers. The electrical contact was always ensured through the TiW adhesion layer, which is not at- 

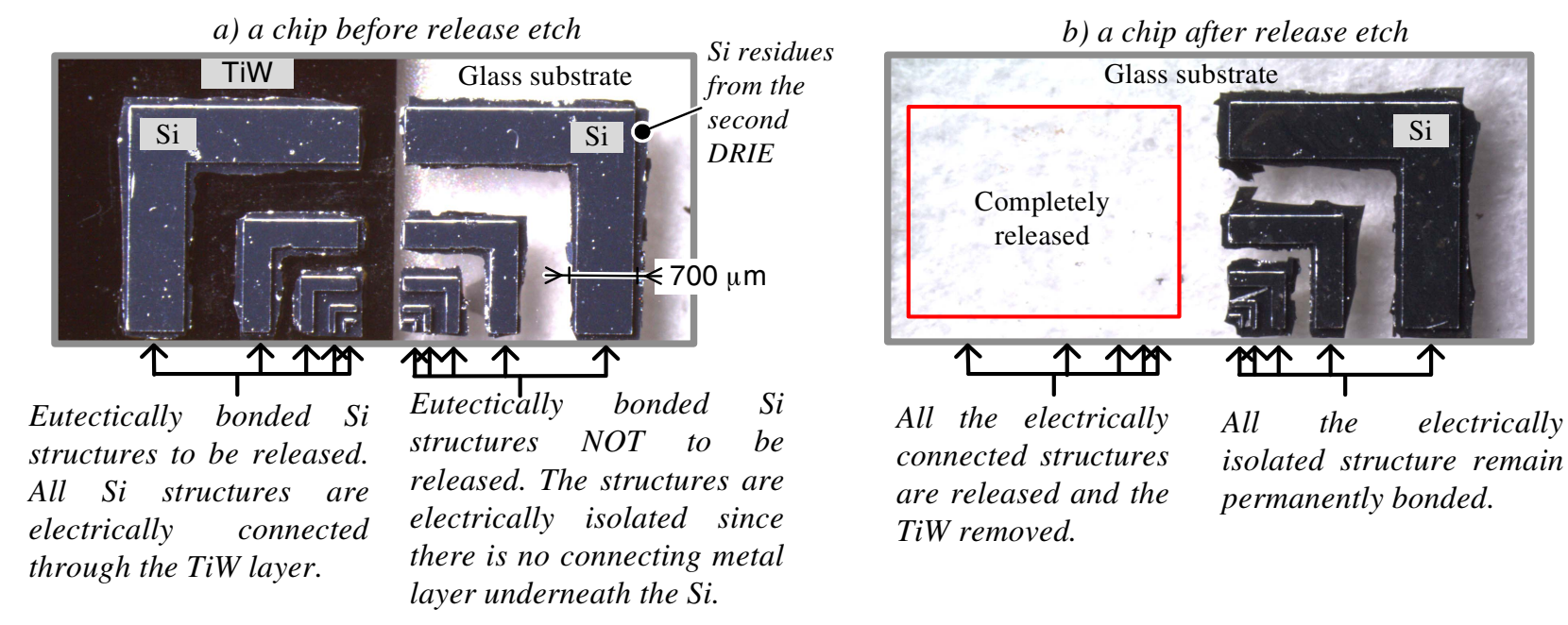

Figure 2: Microscope pictures from the top, showing silicon chips before (a) and after release (b).

tacked by the electrochemical etching.

After the electrochemical etch, the underetched silicon structures were still bonded to the TiW on the substrate. The structures that were electrochemically underetched were completely released after etching the TiW adhesion layer in $\mathrm{H}_{2} \mathrm{O}_{2}$ at room temperature in $1.5 \mathrm{~h}$.

\section{RESULTS AND DISCUSSION}

Figure 2 shows photographs of chips before and after selective etching, illustrating the release of selected structures. In Figure 2a the structures to the left are all electrically connected through the TiW. After the release all the structures as well as the TiW adhesion layer are removed while non of the electrically isolated structures have been removed (Fig. 2b).

One hour of electrochemical etching followed by 1.5 hours of TiW etching in $\mathrm{H}_{2} \mathrm{O}_{2}$ is enough to release a $350 \mu \mathrm{m}$ wide silicon structure. In comparison, silicon structures of the same size that where not electrochemically underetched remained bonded after more than 20 hours in $\mathrm{H}_{2} \mathrm{O}_{2}$.

In contrast to other release etch methods, the only chemicals needed to perform this release are the non harsh sodium chloride solution and the high process compatible $\mathrm{H}_{2} \mathrm{O}_{2}$ [8].

The bond interface was studied to better understand the mechanism of the release i.e. how the electrochemical etching works and why the final TiW etching is needed.

Figure 3 presents a schematic closeup of the bond interface, showing the mechanism of the release as indicated by the observed results.

Before the wafer-bonding, one substrate features a gold layer directly onto silicon and the other substrate features a gold layer on a TiW layer (Fig. 3a). During the bonding, above $363{ }^{\circ} \mathrm{C}$, the two gold layers and parts of the silicon turn into a liquid and the two substrates are unified.
After the bonding the bond interface consists of a solid gold-silicon alloy (Fig. 3b). A SEM picture of the bond interface can be seen in Figure 3e. The bond interface contains silicon rich regions and gold rich regions. This uneven distribution of gold and silicon enables removal of the gold rich regions electrochemically.

During the electrochemical etching, the gold regions around the silicon regions are removed and the silicon rich areas are left, forming a porous layer (Fig. 3c). This porous layer is shown in the SEM picture Figure $3 \mathrm{f}$.

After the removal of the gold the porous silicon is still partly bonded to the TiW layer. To completely release the silicon structures the TiW layer underneath must be removed (Fig. 3d). Due to the porous silicon layer formed, the underetch rate is much faster compared to non electrochemically etched structures. This ensures the selectivity of the release.

In the case that the end application needs a flat surface, a possibility could be to perform a short silicon etch step to remove the porous silicon layer.

\section{CONCLUSIONS}

A novel way of selective release of microstructures has been successfully developed and implemented. This method combines the following advantages: both a strong permanent bond and a temporary bond is achieved on the same die, the footprint of the temporary bonded structures is allowed to be larger than the footprint of the permanent bonded structures and the used etchants offer larger process compatibility than the etchants of other release etch methods.

\section{ACKNOWLEDGEMENT}

This work is part of the Q2M project and receives research funding from the European Commission through the 6th Framework Programme. 

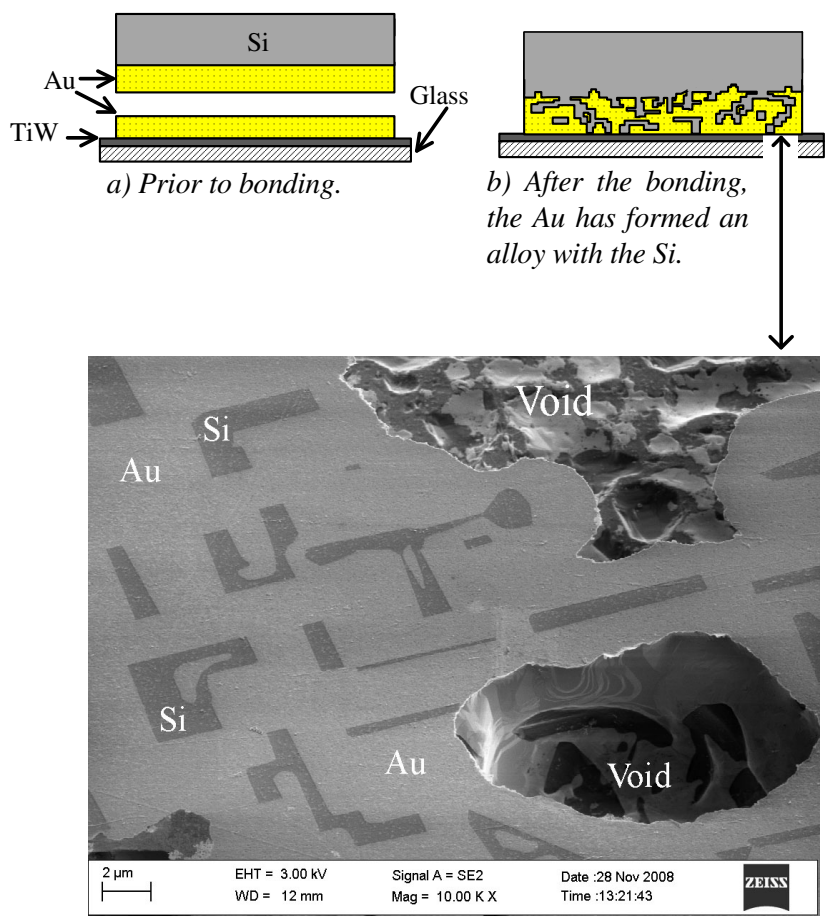

e) SEM picture of the bond interface towards the TiW without doing an electrochemical etch. Au rich regions (>90 atomic \% Au) surround Si rich regions (>90 atomic \% Si). (In this bond interface there are also voids present, i.e. non bonded regions.)

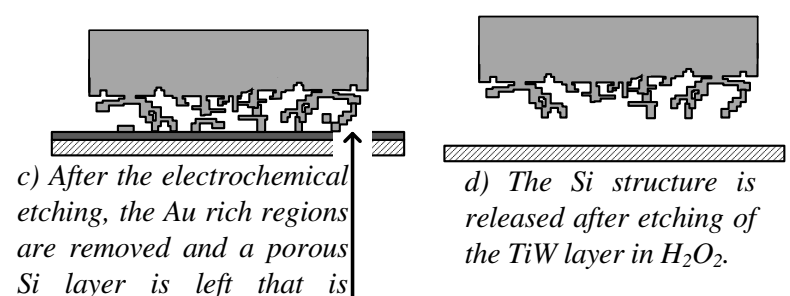

Si layer is left that is

slightly bonded to the TiW. $\downarrow$

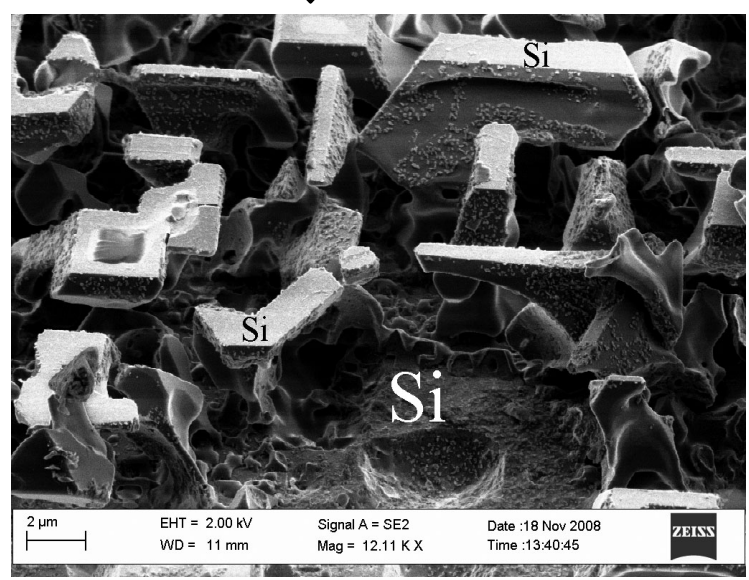

f) SEM picture of the bond interface after an electrochemical etch of the bond interface material. The Au rich regions have been removed and the Si rich regions are left, forming a porous Si layer.

Figure 3: A schematic close up of the bond interface showing the mechanism of the release as indicated by the observed results.

\section{REFERENCES}

[1] F. Niklaus et al., "Adhesive wafer bonding," Journal of Applied Physics, vol. 99, no. 3, p. 031101, February 2006.

[2] J. Oberhammer et al., "Selective wafer-level adhesive bonding with benzocyclobutene for fabrication of cavities," Sensors and Actuators A: Physical, vol. 105, no. 3, pp. 297 - 304, 2003.

[3] T. Veenstra et al., "Use of selective anodic bonding to create micropump chambers with virtually no dead volume," Journal of the Electrochemical Society, vol. 148, no. 2, pp. 68 - 72, 2001.

[4] Y. Cheng et al., "Localized silicon fusion and eutectic bonding for mems fabrication and packaging," Journal of Microelectromechanical Systems, vol. 9, no. 1, pp. 3 $-8,2000$.

[5] S. S. Nasiri et al., "Method and system of releasing a mems structure," United States Patent 7250353, 2007.

[6] O. Gigan et al., "Fabrication and characterization of resonant soi micromechanical silicon sensors based on drie micromachining, freestanding release process and silicon direct bonding," in Proceedings of the SPIE, vol. 4936, USA, 2002, pp. $194-204$.
[7] N. Somjit et al., "Novel concept of microwave mems reconfigurable $7 \times 45^{\circ}$ multi-stage dielectricblock phase shifters," in Proceedings of MEMS, 2009.

[8] K. Williams et al., "Etch rates for micromachining processing-part ii," Journal of Microelectromechanical Systems, vol. 12, no. 6, pp. 761 - 78, 2003.

[9] R. Guerre et al., "Selective transfer technology for microdevice distribution," Journal of Microelectromechanical Systems, vol. 17, no. 1, pp. 157 - 165, 2008.

[10] R. Wolffenbuttel et al., "Low-temperature silicon wafer-to-wafer bonding using gold at eutectic temperature," Sensors and Actuators A (Physical), vol. A43, no. 1-3, pp. $223-9,1994$.

[11] M. Sterner et al., "Maskless selective electrochemically assisted wet etching of metal layers for $3 \mathrm{~d}$ micromachined soi rf mems devices," in Proceedings of MEMS, Jan. 2008, pp. 383-386.

[12] S. Metz et al., "Partial release and detachment of microfabricated metal and polymer structures by anodic metal dissolution," Journal of Microelectromechanical Systems, vol. 14, no. 2, pp. 383 - 391, 2005.

\section{CONTACT}

* H. Gradin, tel: +46-8-790-6284; hegra@ee.kth.se 